\title{
Effect of Hypoxia on Stemness and Differentiation of Dental Pulp Derived Stem Cells
}

\author{
Anupama Kakkar ${ }^{1}$, Puneet Sharma ${ }^{2}$, Manimuthu Mani Sankar ${ }^{1}$, \\ OP Kharbanda ${ }^{3}$, Sujata Mohanty ${ }^{1}$ \\ ${ }^{I}$ Stem Cell Facility, All India Institute of Medical Sciences, New Delhi, India \\ ${ }^{2}$ Department of Biotechnology, All India Institute of Medical Sciences, New Delhi, India \\ ${ }^{3}$ Department of Orthodontics and Dentofacial Deformities, Centre for Dental Education \& Research all India \\ Institute of Medical Sciences, New Delhi, India
}

\begin{abstract}
Stem Cells are characterized by their properties of self renewal and multipotency. These stem cells are localized inside our body at specialized niches, which are known to have low oxygen tension. Dental pulp tissue found in the middle of the tooth is one such niche which experiences hypoxic conditions due to its anatomical location. However under in-vitro conditions, stem cells are cultured under normoxia condition (21\% $\mathrm{O}_{2}$ concentration). It has been known since decades that low oxygen tension in tissue cultures has positive effect on the cell growth. In this study, we tested this hypothesis on in vitro isolated, expanded, Dental Pulp Stem Cells (DPSCs) grown under low oxygen. The comparative evaluation was done between DPSCs grown in normoxia $\left(21 \% \mathrm{O}_{2}\right)$ and Hypoxia $\left(3 \% \mathrm{O}_{2}\right)$ on the basis of proliferation, aging, maintenance of stemness and differentiation potential. Our study indicates that DPSCs when grown in hypoxia exhibited better renewal capacity, lesser population time and hence decreased differentiation. Thus, this study emphasizes that hypoxic culture conditions is better in vitro culture condition for expansion of DPSCs in large scale and maintenance of their stemness for therapeutic purpose.
\end{abstract}

Keywords: Adult Stem Cells, Dental Pulp Stem cells, Differentiation, Hypoxia, Proliferation

\section{Introduction}

Human body contains several microenvironment referred as Stem-cell (SC) niche inhabited by significant number of stem cells. Dental pulp is one such location situated in the middle of the tooth and has been shown to harbor diverse populations of multipotent stem/progenitor cells [1,2]. DPSCs were first isolated in the year 2000, which mainly resides in the perivascular microenviroment of dental pulp. DPSCs have similar characteristics to bone marrow-derived mesenchymal stem cells (BM-MSCs) [3]. These DPSCs have edge over other adult stem cells because of their non-invasive procurement and ease of access [3]. Recently, it has been found that in vivo stem cells reside in niches with low oxygen tension $[4,5]$. Dental pulp tissue is one such niche experiencing hypoxic conditions due to its anatomical location [6]. Researchers have reported that hypoxia leads to increased proliferation in human bone marrow cells [7], neural stem cells [8], fibroblasts [9] and others including dental pulp cells from various sources [10-14]. Although there is consensus on effect of hypoxia on proliferation [10-14], there is diverse view on effect of hypoxia on differentiation potential. Hypoxia has been reported to enhance as well as suppress the differentiation of MSCs into osteocytes and chondrocytes [15-17].

In this study, we have investigated the effect of hypoxia $\left(3 \% \mathrm{O}_{2}\right)$ on proliferation and differentiation potential of stem cells. DPSCs were obtained from impacted third molar and were grown under hypoxia and normoxia $\left(21 \% \mathrm{O}_{2}\right)$ as explant culture. These were examined on the basis of morphology, growth kinetic analysis, senescence assay, surface marker profile and tri-lineage differentiation potential.

\subsection{Sample Collection}

\section{Materials and Methods}

This study was approved by Institutional Committee for Stem Cell Research and Therapy (IC-SCRT). Ten impacted third molars were obtained from 10 individuals (age ranged between 16-18years) who required it to be removed for orthodontic treatment at Department of Orthodontics and Dentofacial Deformities, Centre for Dental Education and Research (CDER), All India Institute of Medical Sciences (AIIMS). Written informed consent was obtained either from the individuals or their legal representative. Following standard tooth extraction procedure, sample was transported to the Stem Cell Facility, AIIMS in cold transport media containing 1× Dulbecco's Modified Eagle's Medium- Low Glucose (DMEM-LG) (Life Technologies, USA), $200 \mu \mathrm{g} / \mathrm{ml}$ of penicillin and $200 \mathrm{U} / \mathrm{ml}$ of streptomycin (Life Technologies, USA) and 1X antimycotic (Life Technologies, USA) at $4^{\circ} \mathrm{C}$. The tooth samples were processed immediately for the establishment of primary culture. 


\subsection{Processing, isolation and establishment of DPSCs culture}

Any extraneous soft connective tissue was removed from the outer surface of the tooth using needle or sterile scalpel blade. It was then cracked opened with 7" heavy duty bone cutter and pulp was carefully removed using sterile fine forceps and needle. The pulp was rinsed twice with 1X PBS containing antibiotics for 20 minutes. It was then cut into small pieces $(<1 \mathrm{~mm})$ and explant culture were seeded into two $35 \mathrm{~mm}$ Petri dishes (Becton Dickinson, USA) with DMEM-LG containing 10\% Fetal Bovine Serum (FBS) (HyClone, USA), penicillin $(100 \mathrm{U} / \mathrm{ml})$ and streptomycin $(100 \mu \mathrm{g} / \mathrm{ml})$. One of the $35 \mathrm{~mm}$-petri dish was incubated at $37^{\circ} \mathrm{C}$ in humidified atmosphere containing $21 \% \mathrm{O}_{2}$ and $5 \% \quad \mathrm{CO}_{2}$ and the second dish was incubated at $37^{\circ} \mathrm{C}$ in humidified atmosphere containing $3 \% \mathrm{O}_{2}$ and $5 \% \mathrm{CO}_{2}$. After 3 days, the media was changed and the subsequent media changes were done at every third day taking care not to dislodge the explants. After establishment of DPSC primary cultures of $70 \%-80 \%$ confluent, the cells were trypsinized using $0.25 \%$ trypsin-EDTA (TE) (Life-Technologies, USA) followed by incubation for $2-3$ minutes at $37^{\circ} \mathrm{C}$. Cells were split in $1: 3$ ratio and incubated at $37^{\circ} \mathrm{C}, 5 \% \mathrm{CO}_{2}$ and respective $\mathrm{O}_{2}$ concentration with media change on every third day.

\subsection{Culture Conditions}

Hypoxic culture conditions were maintained by culturing of DPSCs (hereafter referred as DPSC-H) in $3 \% \mathrm{O}_{2}, 5 \% \mathrm{CO}_{2}$, and $92 \% \mathrm{~N}_{2}$ in a $95 \%$ humidified atmosphere. Control cells were kept under normoxic conditions (hereafter referred as DPSC-N) $\left(21 \% \mathrm{O}_{2}\right) .[18]$

\subsection{Characterization of DPSCs}

\subsubsection{Morphological Analysis}

Both DPSC-H and DPSC-N were regularly viewed under Phase Contrast Inverted Microscope (Nikon 80i, Japan) for any morphological changes. Any signs of contamination were also monitored.

\subsubsection{Immunophenotyping}

Immunophenotype of cultured DPSCs $(\mathrm{N}=10)$ was determined by flow cytometry. DPSC-H and DPSC-N at passage 3 were harvested using 1X TrypLE Express (Life Technologies, USA). Single cell suspension of $1 \times 10^{6}$ cells $/ \mathrm{ml}$ was prepared and from this $100 \mu \mathrm{L}$ of cell suspension $\left(1 \times 10^{5}\right.$ cells $)$ were incubated with anti-human antibodies CD73-Phycoerythrin (PE), anti-CD90- Phycoerythrin conjugated with Cyanine dye derivative (PECy5), anti-HLA Class I-Allophycocyanin (APC), anti-HLA Class II- Fluorescein Isothiocyanate (FITC) (Becton Dickinson, USA), anti-CD29- FITC and anti-CD105- APC (eBioscience, USA) at room temperature in dark for 1 hour. Unstained cells were included as controls. The cells were acquired in LSR II flow cytometer (Becton Dickinson, USA) with at least 5000 events for each sample and analyzed with FACs DIVA software (version 6.1.2). [19]

\subsubsection{Measurement of Metabolic Activity by MTT Assay}

Proliferation rate of DPSCs $(\mathrm{N}=5)$ was measured by 3-(4, 5-Dimethylthiazol-2-yl)-2, 5diphenyltetrazolium bromide (MTT) assay. DPSC-N and DPSC-H passage three cells were seeded in triplicates onto 96 well flat bottom plate at a concentration of $5 \times 10^{3}$ cells per well. On days 1, 3, 6, 9, 12, 15, 22 and 28 , media was replaced with $180 \mu \mathrm{l}$ fresh media and $20 \mu \mathrm{l}$ MTT reagent $(5 \mathrm{mg} / \mathrm{ml}$ in PBS) was added and further incubated for 4 hours at $37^{\circ} \mathrm{C}$ in respective incubating conditions. The supernatant media was removed carefully and $150 \mu \mathrm{l}$ DMSO (Sigma, USA) was added to each well to dissolve formazan crystals and incubated at $37^{\circ} \mathrm{C}$ for $30 \mathrm{~min}$ in dark. The optical density (OD) was measured at $570 \mathrm{~nm}$ and $660 \mathrm{~nm}$ by spectrophotometer (BioTek, USA).

\subsubsection{Proliferation Assay}

DPSCs for each sample ( $\mathrm{N}=5)$ were seeded at a density of $25 \times 10^{3}$ cells per $35 \mathrm{~mm}$ petri dish (Becton Dickinson, USA) and grown under normoxia and hypoxia condition as mentioned earlier. After 3 days, DPSC-H and DPSC-N were harvested, enumerated and assessed for viability using Trypan Blue dye exclusion assay. The PDT was obtained by the formula [20]:

$\mathrm{PDT}=\mathrm{T}-\mathrm{To} \log 2(\log \mathrm{N}-\log \mathrm{No})$

Where, T: Time of harvesting

To: Time of seeding

$\mathrm{N}$ : Number of cells harvested

No: Number of cells seeded

\subsubsection{Senescence Assay}


In order to evaluate senescence, $\beta$-galactosidase staining was performed using commercially available kit (Cell Signalling Technology, USA) according to manufacturer's protocol. Briefly, DPSC-N and DPSC-H of $3^{\text {rd }}$ passage $\left(\mathrm{N}=5\right.$ each) were seeded in duplicates and incubated in respective $\mathrm{O}_{2}$ concentration until they become confluent (75-80\%). Cells were washed with 1XPBS and fixed with $2 \%$ formaldehyde/0.2\% glutaraldehyde solution for 15 minutes. Followed by two washes with PBS $\beta$-galactosidase chromogenic substrate solution containing $1 \mathrm{mg} / \mathrm{ml}$ 5-bromo-4-chloro-3-indolyl- $\beta$-galactosidase (X-Gal), 40mM citric acid ( $\mathrm{pH}$ 6.0), $5 \mathrm{mM}$ potassium ferrocyanide, $5 \mathrm{mM}$ potassium ferricyanide, $150 \mathrm{mM} \mathrm{NaCl}$ and $2 \mathrm{mM} \mathrm{MgCl}_{2} \mathrm{was}$ added. The petri dishes were then incubated at $37^{\circ} \mathrm{C}$ overnight. Percentage of cells expressing $\beta$-galactosidase was measured in three random fields under inverted phase contrast microscope (Nikon 80i, Japan) [20].

\subsubsection{Tri-lineage Differentiation of DPSCs}

DPSC-H and DPSC-N of passage 3 ( $\mathrm{N}=5$ each) were used for tri-lineage differentiation. Undifferentiated cells were used as experimental controls.

\subsubsection{Osteogenic Differentiation}

DPSC-H and DPSC-N were induced with DMEM-LG (Life Technologies, USA) supplemented with $10 \%$ FBS (Life Technologies, USA), $50 \mu \mathrm{M}$ ascorbic acid-2- phosphate, $0.1 \mu \mathrm{M}$ dexamethasone and $10 \mathrm{mM} \beta$ glycerophosphate (Sigma-Aldrich, USA) for 28 days. Osteogenic differentiation was confirmed by Alizarin Red S staining (Himedia, India).

\subsubsection{Adipogenic Differentiation}

For adipogenic differentiation, DPSC-H and DPSC-N were cultured in DMEM- LG with 10\% FBS supplemented with $100 \mu \mathrm{M}$ Indomethacin, $1 \mu \mathrm{mol} / \mathrm{L}$ dexamethasone, 500 $\mu \mathrm{M}$ 3-isobutyl-1-methylxanthine (IBMX), $1 \mu \mathrm{g} / \mathrm{ml}$ Insulin (Sigma-Aldrich, USA) for 21 days. Media was changed every third day for 28 days. The differentiation was confirmed by Oil Red O staining (Himedia, India) [19].

\subsubsection{Chondrogenic Differentiation}

Commercially available kit (Life Technologies, USA) for chondrogenic differentiation was used. Briefly, micromass culture was generated by seeding $10 \mu \mathrm{l}$ droplets (consisting about $1 \times 10^{5}$ cells) of DPSC-H and DPSC-N cell solution in $35 \mathrm{~mm}$ petri dish and incubated for 2 hours at $37^{\circ} \mathrm{C}$ with $5 \% \mathrm{CO}_{2}$ in respective oxygen concentration followed by addition of chondrogenic differentiation media for 21 days. The differentiation was confirmed by Alcian Blue staining (Sigma-Aldrich, USA).

\subsubsection{Reverse Transcriptase-PCR (RT-PCR)}

DPSCs-H and DPSCs-N (osteo and chondro induced cells, $\mathrm{N}=5$ each) were lysed using TRIzol (SigmaAldrich, USA) and RNA was isolated using Phenol-Chloroform method. cDNA was prepared using $1 \mu \mathrm{g}$ of RNA by Reverse transcriptase (RT) enzyme (Promega, USA). RNA was reverse transcribed by mixing $1 \mu \mathrm{g}$ of RNA with $0.5 \mu \mathrm{g}$ of oligo-dT followed by 5 minutes incubation at $70^{\circ} \mathrm{C}$. Final volume was made $20 \mu \mathrm{l}$ by further addition of RT-Buffer (1X), 200U M-MLV RT, 2mM dNTPs and 40U RNasin plus RNase inhibitor (all Promega, USA). Reaction mixture was incubated at $37^{\circ} \mathrm{C}$ for 1 hour. For confirmation of osteogenic and chondrogenic differentiation, expression profile of osterix and collagen- II was checked by semi-quantitative RT-PCR respectively and Glyceraldehyde-3-phosphate dehydrogenase (GAPDH) was used as housekeeping gene (BioRad, USA).

Table 1 Primer Sequences and Reaction Conditions for PCR Sequence

\begin{tabular}{|c|c|c|c|c|}
\hline S. No & Gene & Sequence & Annealing $\left({ }^{\circ} \mathbf{C}\right)$ & Size (bp) \\
\hline 1 & GAPDH & $\begin{array}{c}\text { Forward:5'-GAG TCA ACG GAT TTG GTC GT-3' } \\
\text { Reverse: 5'-GAC AAG CTT CCC GTT CTC AG-3' }\end{array}$ & 56 & 200 \\
\hline 2 & Osterix & $\begin{array}{c}\text { Forward: 5'-GGC ACA AAG AAG CCG TAC TC-3' } \\
\text { Reverse: 5'-CAC TGG GCA GAC AGT CAG AA-3' }\end{array}$ & 57 & 284 \\
\hline 3 & $\begin{array}{c}\text { Collagen- } \\
\text { II }\end{array}$ & $\begin{array}{c}\text { Forward: 5'-ACC AAA GGG ACA GAA AG-3' } \\
\text { Reverse: 5'-CAG CTT CAC CAT CAT CAC C-3' }\end{array}$ & 56 & 400 \\
\hline
\end{tabular}

\subsubsection{Statistical Analysis}

All values were stated as mean \pm standard deviation (SD). Student's t test was applied for MTT assay and Population doubling time. Two-sample Wilcoxon rank-sum (Mann-Whitney) test was used for Flow cytometry- and senescence assay- Fisher's exact test. The degree of significance was set at $\mathrm{p}<0.05$.

\section{Results}




\subsection{Establishment of DPSC Primary Cultures}

Initial migration of cells from explants was observed within 6-7 days of culture among DPSC-H where as in DPSC-N migration was observed in 10-12 days (Fig 1). DPSC-H cells established monolayer of 70-80\% confluence in 25-30days while DPSC-N took 6-7days more to establish monolayer with similar confluence. Cells were passaged once they reached $70-80 \%$ confluency.
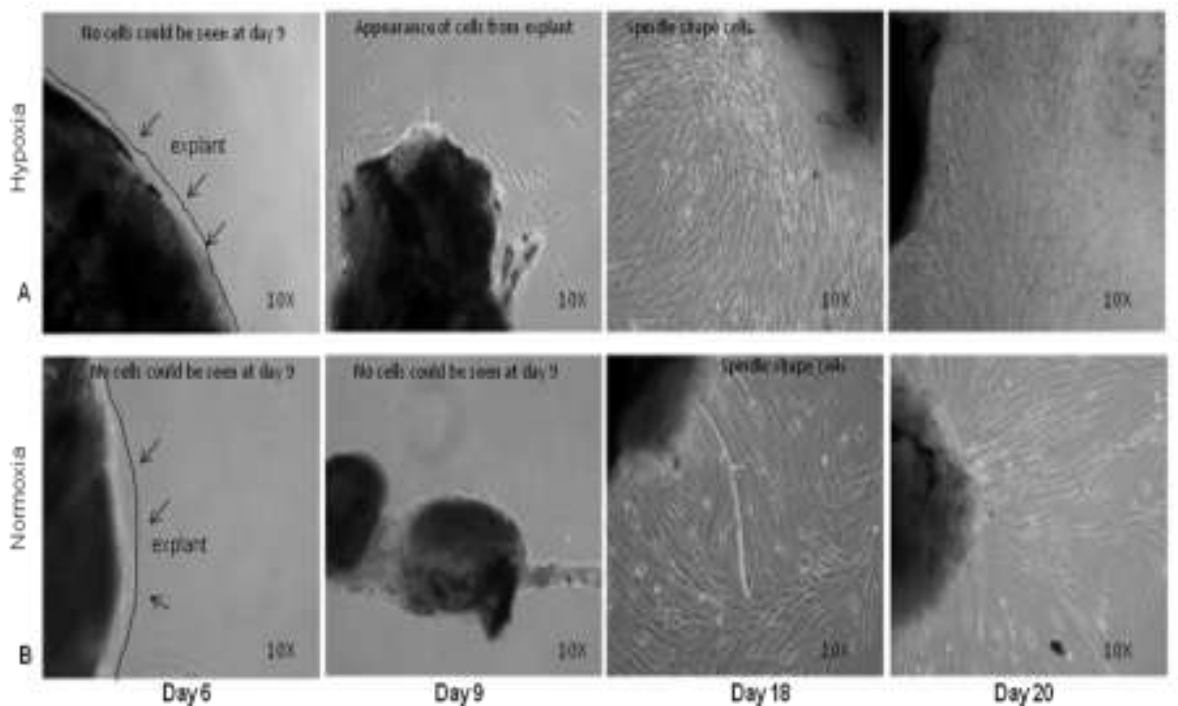

Figure 1 Explant culture of DPSCs from Dental Pulp

DPSCs (10X magnification) migrating out of explants while grown in (A) Hypoxia and (B) Normoxia from day 6 to day 20.

\subsection{DPSCs Morphology}

DPSC-N and DPSC-H both exhibited fibroblast like spindle shaped morphology and expanded in whirlpool like manner bone marrow derived mesenchymal stem cells (BM-MSCs) (Fig 2). During the long term cultivation no visual signs of culture degeneration or spontaneous differentiation could be observed in both the groups.
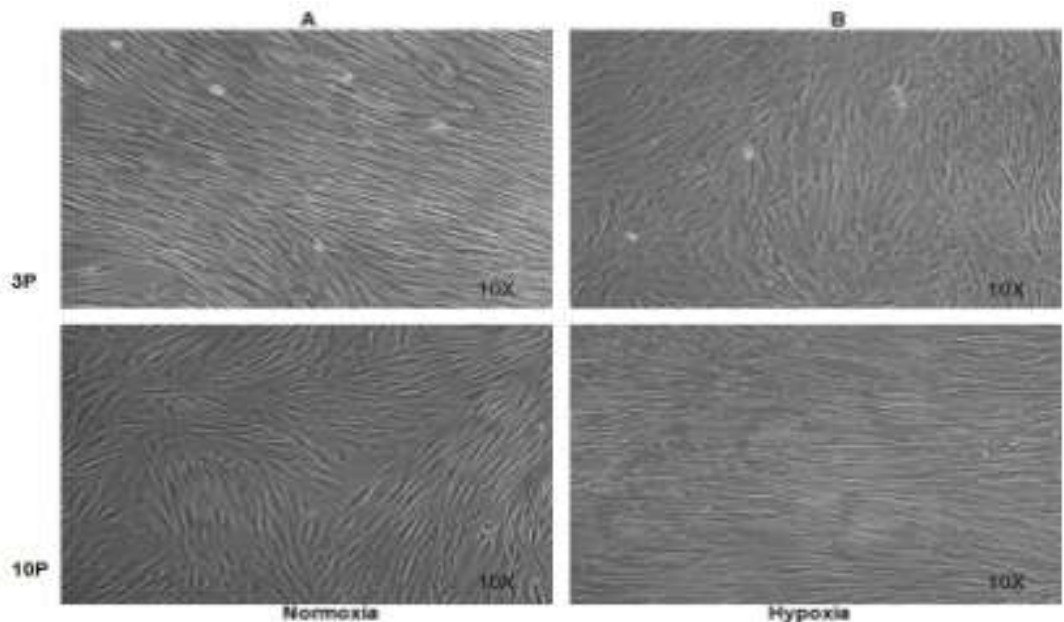

Figure 2 Morphological characteristics of DPSCs

Fibroblast like morphology of DPSCs at early (passage 3) and late (passage 10) passage when grown in (A) Normoxia (B) Hypoxia under phase contrast microscope (10X).

\subsection{Surface Marker Profiling}

Both DPSC-H and DPSC-N groups were found to be positive for CD29, CD73, CD90, CD105, HLA-I and negative for HLA II and CD34/45. However, no significant difference was observed ( $p=0.796)$ (Fig 3). 


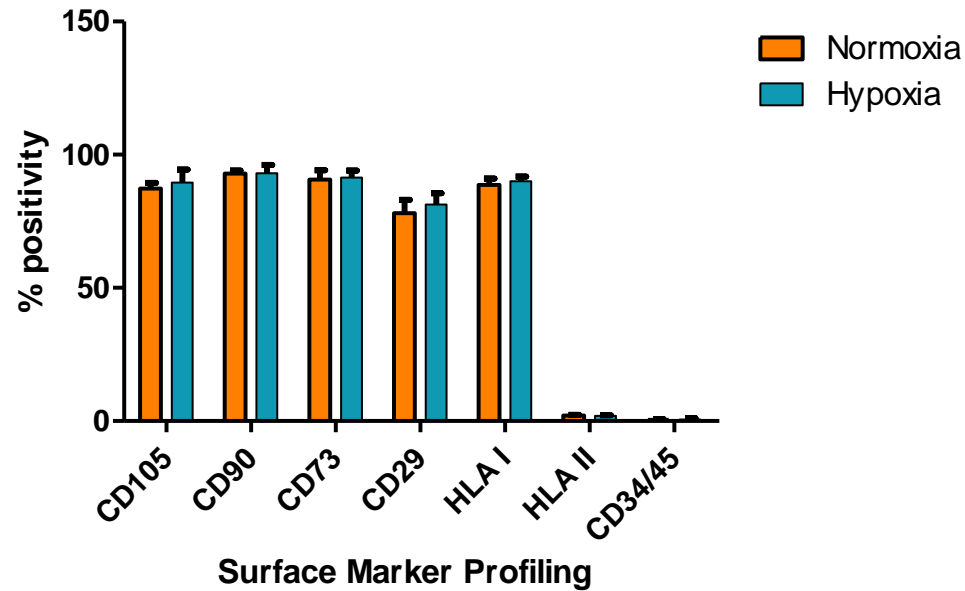

Figure 3 Surface Marker Profiles of DPSCs

\subsection{Growth Kinetics}

To detect the effect of hypoxic condition on DPSCs proliferation MTT assay was performed over a span of 28 days. On all assay days cell proliferation in hypoxic cultures was greater than that of normoxic group. However, statistically significant difference in proliferation rate was observed only on assay 1, 6, 12 and 22 days (Fig 4A).

Furthermore, PDT for hypoxic group was $26.75 \pm 2.76$ hours while the normoxic group had significantly longer doubling time of $38.59 \pm 4.25$ hours (Fig 4B).

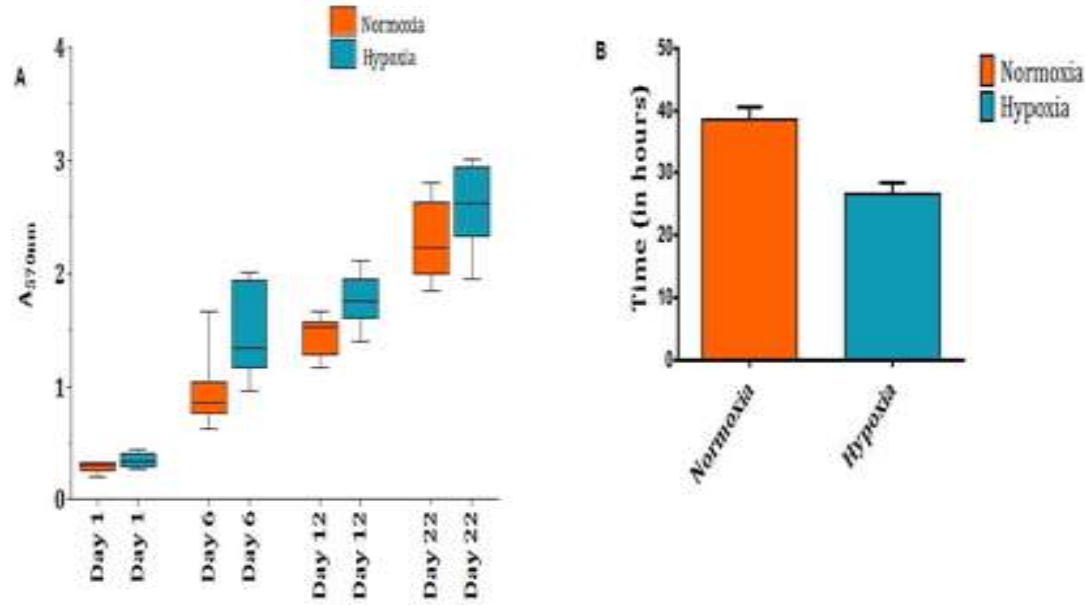

Figure 4 Proliferation patter and Population Doubling Time of DPSCs

(A) MTT assay depicting proliferation rate of DPSCs and (B) Comparison of Population Doubling Time under Normoxia and Hypoxia.

\subsection{Senescence Assay}

It was observed that at $10^{\text {th }}$ passage DPSCs-H and DPSCs- $\mathrm{N}$ exhibited senescence in $0.5 \%$ and $1 \%$ of cells, respectively (Fig 5). 

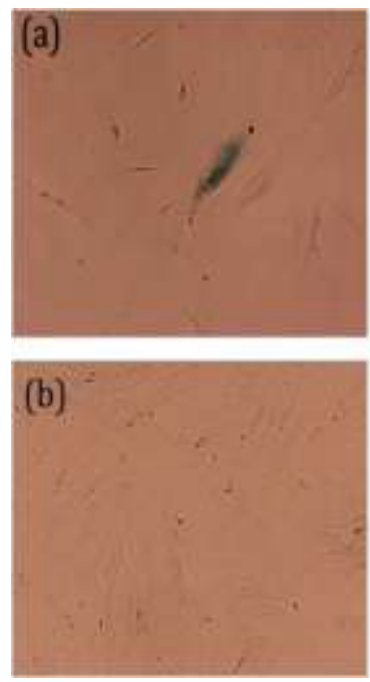
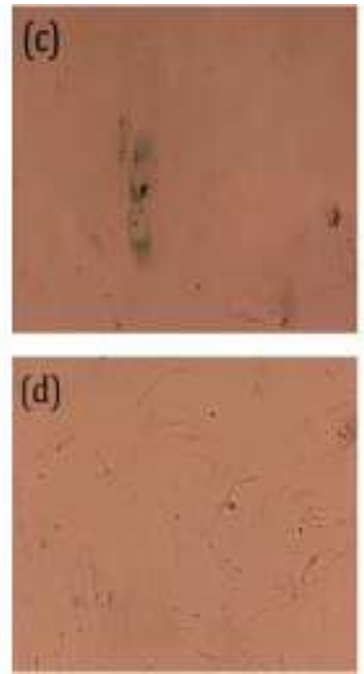

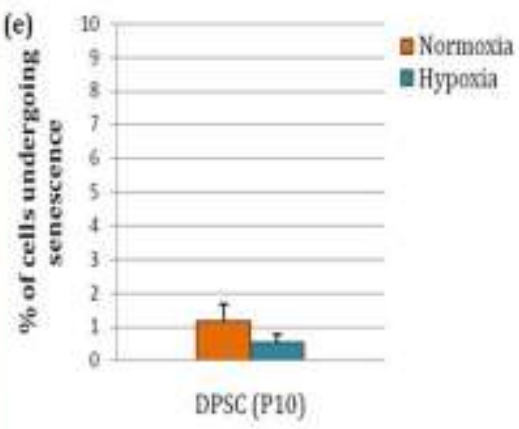

Figure 5 Senescence Rate in DPSCs

Microscopic observation (10X) of DPSCs grown under Hypoxia (a, b) and Normoxia (c, d). Graphical representation of $\%$ of cells found positive for senescence in two groups (e).

\subsection{Tri-lineage Differentiation Potentials}

DPSCs cultured in hypoxic and normoxic conditions were able to differentiate towards osteogenic lineage. Controls grown under normoxic and hypoxic condition during the experimental period did not show any spontaneous induction; rather the cells grew in confluency and resulted in confluency. Osteoinductive cultures of DPSC-N and DPSC-H stimulated for four weeks, exhibited osteogenic nodule formation (Fig. $6 \&$ 7) and chondrogenic lineages (Fig. $8 \&$ 9) after four weeks of culture in appropriate differentiation medium. Uninduced DPSC-H and DPSC-N did not show any visual signs of spontaneous differentiation during the experimental period of four weeks. This was confirmed by their Alizarin Red S and Alcian Blue staining. On an average, osteogenic nodules took about 15-16 days to develop in normoxia condition while in hypoxia it took them about 16-18 days. Similar trend was observed for chondrogenic differentiation where chondrogenic nodules in normoxic condition took 3-4 days to develop while in hypoxic condition they took 6-7 days. These differentiations were further successfully confirmed using RT- PCR detection of osterix (osteogenic marker) (Fig.7) and collagen-II (chondrogenic marker) (Fig. 9). However, we were not able to achieve adipogenic differentiate in any of our DPSC-N and DPSC-H cells (Fig.10).

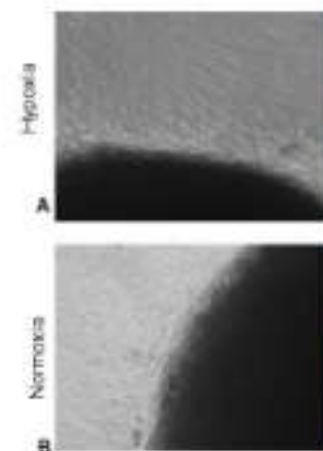

(i)
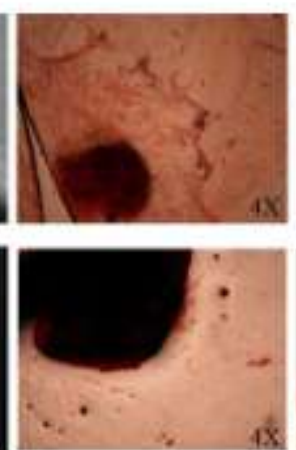

(ii)
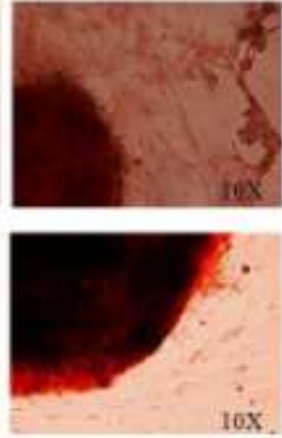

(iii)
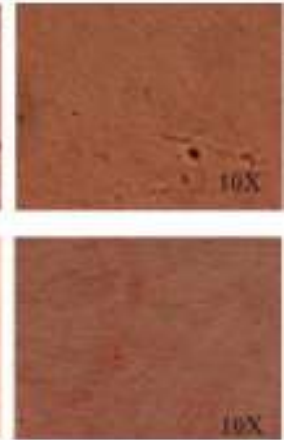

(iv)

Figure 6 Alizarin Red S Staining for confirmation of Osteogenic differentiation

Representative images showing Osteoinductive cultures of DPSCs under Hypoxia (A) and Normoxia(B) indicated the deposition of mineralized matrix within the osteogenic nodules as confirmed with Alizarin Red S staining. Bright field images of induced cultures at 10x (i), Alizarin Red S staining at 4x and 10x (ii, iii) uninduced cells at 10x (iv). 

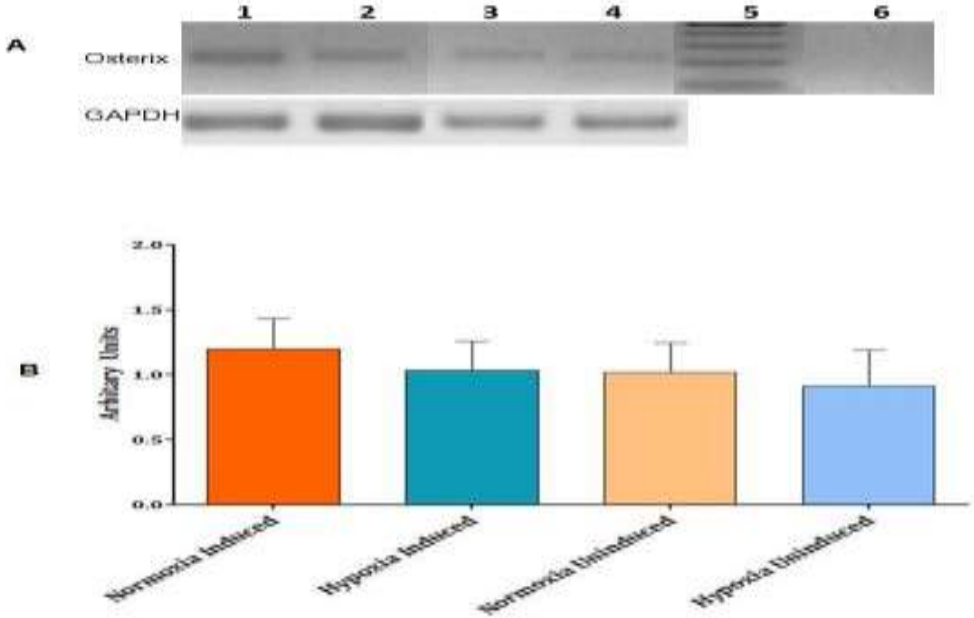

Figure 7 mRNA expression of Osterix for confirmation of Osteogenic differentiation

(A) Agarose gel electrophoresis image (Lane 1: Induced DPSC-N, Lane 2: Induced DPSC-H, Lane 3: uninduced DPSC-N, Lane 4: uninduced DPSC-H, lane 5: Ladder, Lane 6: negative control. Normalization of each band obtained was done with respect to its GAPDH. (B) Densitometry pattern of osterix in induced cultures under Hypoxia and Normoxia.

(B)
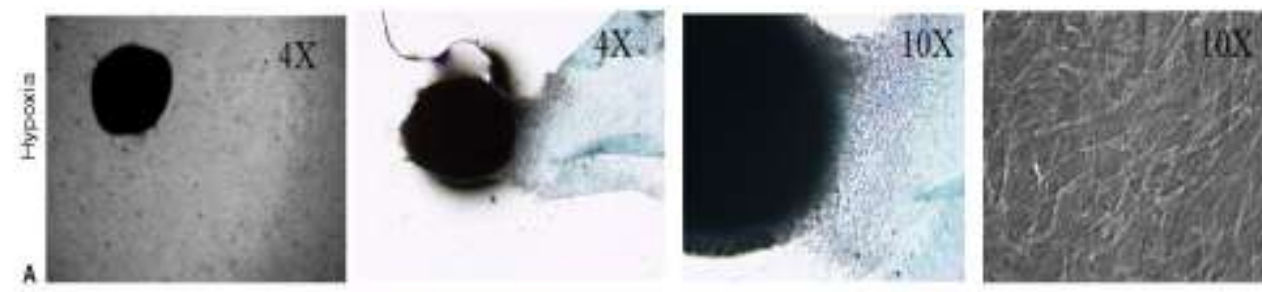

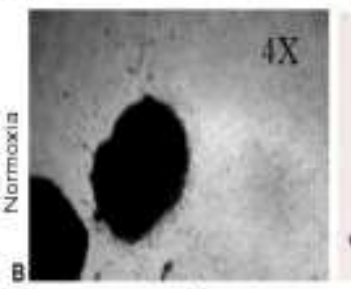

(i)

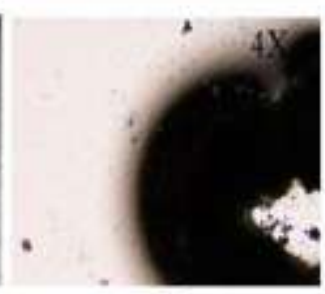

(ii)

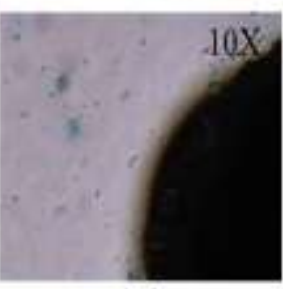

(iii)

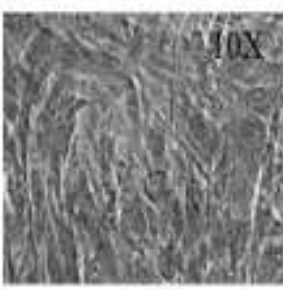

(iv)

Figure 8 Alcian Staining for confirmation of Chondrogenic differentiation

Representative images showing Chondrogenic cultures of DPSCs under Hypoxia (A) and Normoxia(B) Bright field images of induced cultures at 4x(i), Alcian Blue staining staining at $4 \mathrm{x}$ and 10x (ii, iii), uninduced cells at 10x (iv).
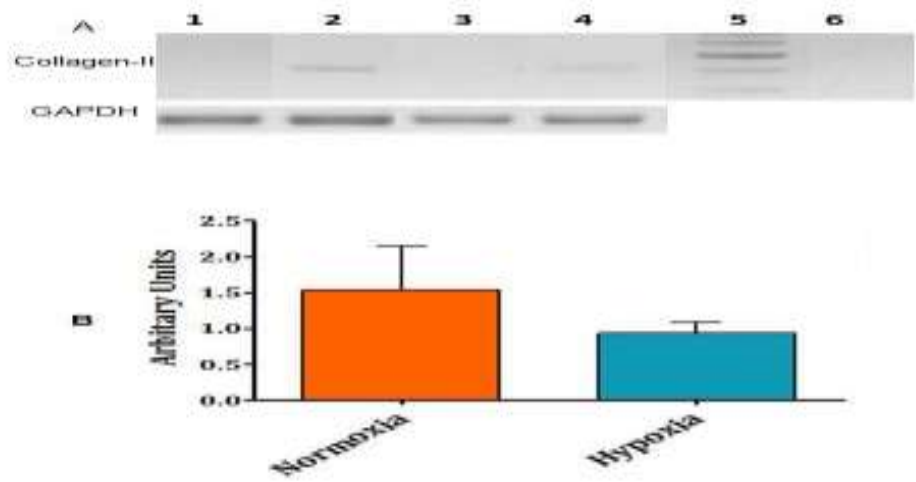
Figure 9 mRNA expression of Collagen-II for confirmation of Chondrogenic differentiation

(A) Agarose gel electrophoresis image (Lane 1: Uninduced DPSC-N, Lane 2: Induced DPSC-N, Lane 3: uninduced DPSC-H, Lane 4: Induced DPSC-H, lane 5: Ladder, Lane 6: negative control. Normalization of each band obtained was done with respect to its GAPDH. (B) Densitometry pattern of collagen-II in induced cultures under Hypoxia and Normoxia.
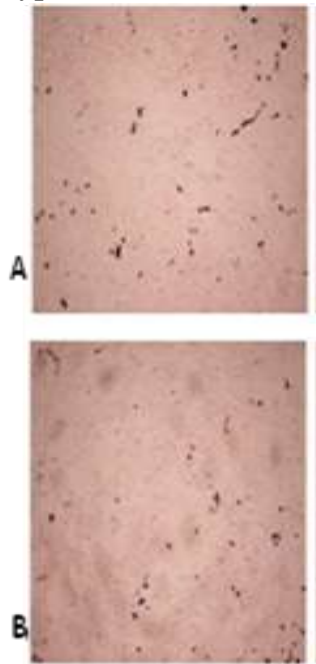

(i)
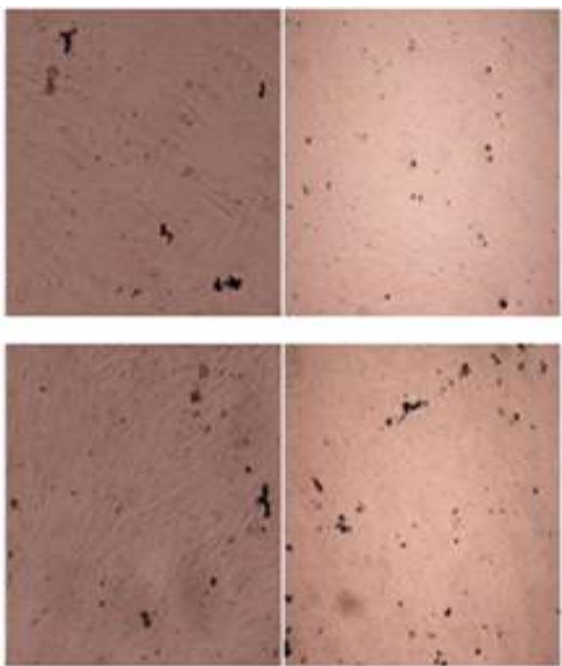

(ii)
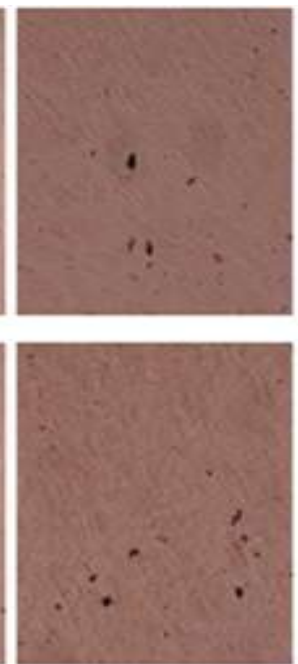

(iv)

Figure 10 Oil Red O Staining for confirmation of Adipogenic differentiation

Representative images showing adipogenic cultures of DPSCs under Hypoxia (A) and Normoxia(B) Bright field images of induced cultures at $4 x$ and 10x (i,ii), uninduced cells at $4 x$ and 10x (iii, iv). None of the samples induced (A and B) showed Oil Red O stained lipid globules inside the cells.

\section{Discussion}

During the last few decades stem cells have been derived from various tissue sources including dental pulp. DPSC are now being recognized as vital source in regenerative dentistry. Due to their easy availability and multipotency characteristics, DPSCs might have clinical implication beyond dentistry. Expansion of these cells while preserving their stem cell characteristics or stemness is of prime importance for their successful use in clinical practices. Among all factors, hypoxia has been shown to have positive effect on cell proliferation [21].

In the current study, we demonstrated the effect of hypoxia on DPSCs proliferation, stemness, differentiation and aging. We could isolate healthy, spindle shaped cells using explants method in both hypoxia and normoxia These were cultured and expanded successfully in DMEM-Low Glucose (LG) media with 10\% FBS which is in sync with previous reports $[22,23]$. However, Govindasamy et al, observed low cellular proliferation, higher senescence rate and abnormal morphology in DPSCs cultivated in DMEM-LG [24]. Also, Lizier et al, demonstrated MEM- $\alpha$ and DMEM/F12 as most appropriate media for DPSCs expansion [25]. Hence, we have shown that DMEM-LG is the simple media that can be used for successfully isolation and expansion of DPSCs both in normoxia and hypoxia.

We employed explants culture method for DPSC isolation and got successful isolation in both normoxia and hypoxia. This explant culture method fulfils the criteria of an ideal source of mesenchymal stem cells for clinical application because they are harvested using procedures that have minimal invasiveness and morbidity, and high content of stem cells. Also, this is a simple, gentle, economic and enzyme free method. Time taken for initiation and establishment of culture is lesser in hypoxia as indicated by early emergence of cells spreading out of the explants, which indicates the advantage of hypoxia in scaling up of stem cells for future therapeutic purpose in lesser duration.

There was no morphological difference observed between DPSCs grown in normoxia and hypoxia and they exhibited fibroblast like spindle shaped morphology as reported for BM-MSCs [26]. Surface marker profiling which is hallmark of mesenchymal stem cells, demonstrated that DPSCs of both groups were positive for MSCs markers CD105, CD90, CD73 and CD29 and were negative for hematological markers CD 34/45 which is in sync with other previous studies [27, 28]. Moreover, DPSCs were also found to be positive for immunological marker HLA I and negative for HLA II. Expression of MSCs markers substantiates the fact that these cells have mesenchymal characteristics due to their ecto-mesenchymal origin. Quantitatively, no 
significant difference was observed in the percentage of cells expressing these markers in the two groups under investigation $[29,30]$.

Growth kinetics studies done by MTT showed that DPSCs-H exhibited higher proliferation than DPSC-N for up to 21 days with significant difference and significantly lower PDT as compared to DPSCs-N. These cells could be successfully propagated for prolonged period of time (5 months; 14 to 21 passages for hypoxic cultures and up to 14 passages for normoxic culture) in undifferentiated state without any visual signs of spontaneous differentiation and culture degeneration. This was further confirmed by the expression of surface markers, which were found to be above $85 \%$ up to $15^{\text {th }}$ passage in hypoxia (data not shown), which depicts that hypoxia helps maintain its stem cell characteristics. Also, senescence assay depicted that cells were healthy and about $1 \%$ of cells in normoxia and $0.5 \%$ cells in hypoxia were undergoing senescence though, the difference was not statistically significant which is in line with previous reports [20].

Apart from self renewal, we also checked effect of hypoxia on tri-lineage differentiation potential of DPSCs. In our study, DPSCs successfully differentiated into osteogenic and chondrogenic lineages. Osteogenic differentiation was confirmed by Alizarin red S staining and RT-PCR for Osterix gene. Densitometry analysis of PCR amplified cDNA of osterix from two groups revealed marginally lower expression of this osteogenic marker gene in induced hypoxic culture [7,31]. Similar observation has been recently reported in case of Adipose Stem Cells, where hypoxia was found to inhibit osteogenic differentiation [32,33]. Similarly, Chondrocytes differentiation was confirmed by Alcian blue staining and RT-PCR for Collagen-II. Densitometry analysis for collagen-II from induced samples of two groups revealed decreased expression of this chondrogenic marker in hypoxic culture, which is in sync with earlier report for decreased chondrogenesis of adipose stem cells in hypoxia [32]. Meanwhile contradictory findings have been reported showing enhanced chondrogenesis of MSCs in hypoxia [22,34]. For, adipogenic differentiation, we could not see any adipocytes upon induction in both the groups. These results are in agreement with previous studies where either researcher were not able to differentiate these cells into lipid laden adipocytes or observed very low differentiation using same inducers $[3,20]$, and this difference in degree of differentiation might be their tissue specific behaviour. In 2010 , a study was published suggesting that a more potent adipogenic inductive culture can induce DPSCs to form oil red $\mathrm{O}$ positive lipid containing adipocytes containing hydrocortisone instead of dexamethasone [35]. Similarly, densitometry analysis of PCR amplified cDNA of collagen-II from induced samples of two groups revealed decreased expression of this chondrogenic marker in hypoxic culture, which is in sync with earlier report for decreased chondrogenesis of adipose stem cells in hypoxia [32]. Meanwhile contradictory findings have been reported showing enhanced chondrogenesis of MSCs in hypoxia [22,34].

Role of hypoxia in proliferation and differentiation has been investigated since years. However, we were not able to study the underlying mechanism. Growing literature states the role of Hypoxia Induced Factor (HIF) interaction with notch signaling in maintenance of undifferentiated state [34]. The HIF-1 $\alpha$ is induced after low oxygen exposure in certain cell types [36]. However, in present study we did not study its role in maintaining stemness of the stem cells.

\section{Conclusions}

We demonstrated that DPSCs can be successfully isolated from human third molars using explant method, which is cost effective and enzyme free. These can be cultivated successfully in DMEM-LG $+10 \%$ FBS. Hypoxia i.e. low oxygen tension enhances proliferation, maintains stemness and suppresses tri-lineage differentiation of these cells. Therefore, hypoxia culture may be used for scale up of stem cells required for further therapeutic purpose.

\section{Acknowledgements}

The work was supported by All India Institute of Medical Sciences (AIIMS), New Delhi, Department of Biotechnology-Centre of Excellence (DBT-COE), and Council of Scientific and Industrial Research (CSIR), India.

\section{References}

[1]. Yamamura T, Shimono M, Koike H, Terao M, Tanaka Y, Sakai Y, Inoue T, Yoshiki S, Tachikawa T, Kawahara H, Watanabe O. Differentiation and induction of undifferentiated mesenchymal cells in tooth and periodontal tissue during wound healing and regeneration. The Bulletin of Tokyo Dental College, 21(3), 1980, 181-221.

[2]. Fitzgerald M, Chiego J. D., Heys D. R. Autoradiographic analysis of odontoblast replacement following pulp exposure in primate teeth. Archives of Oral Biology, 35(90), 1990, 707-715.

[3]. Gronthos S., Mankani M., Brahim J., Robey P. G., Shi S. Postnatal human dental pulp stem cells (DPSCs) in vitro and in vivo. Proceedings of National Academy of Science USA, 97(25), 2000, 13625-13630.

[4]. Jing D, Wobus M, Poitz DM, Bornhäuser M, Ehninger G, Ordemann R. Oxygen tension plays a critical role in the hematopoietic microenvironment in vitro. Haematologica, 97(3), 2012, 331-339. 
[5]. Mohyeldin A, Garzón-Muvdi T, Quiñones-Hinojosa A. Oxygen in stem cell biology: a critical component of the stem cell niche. Cell Stem Cell, 7(2), 2010, 150-161.

[6]. Yu CY, Boyd NM, Cringle SJ, Alder VA, Yu DY. Oxygen Distribution and Consumption in Rat Lower Incisor Pulp. Archives of Oral Biology, 47 (7), 2002, 529-536.

[7]. D'Ippolito G, Diabria S, Howard GA, Roos BA, Schiller PC. Low Oxygen Tension Inhibits Osteogenic Differentiation and Enhances Stemness in Human MIAMI Cells. Bone, 39 (3), 2006, 513-522.

[8]. Santilli G, Lamorte GL, Carlessi L, Ferrari D, Nodari LR, Binda E, Delia D, Vescovi AL, Filippis LD. Mild Hypoxia Enhances Proliferation and Multipotency of Human Neural Stem Cells. PLoS ONE, 5(1), 2010, e8575.

[9]. Packer L. and Fuehr K. Low Oxygen Concentration Extends the Lifespan of Cultured Human Diploid Cells. Nature, 267 (5610), 1977, 423-425.

[10]. Agata H, Kagami H, Watanabe N, Ueda M. Effect of ischemic culture conditions on the survival and differentiation of porcine dental pulp-derived cells. Differentiation, 76 (9), 2008, 981-993.

[11]. Amemiya K, Kaneko Y, Muramatsu T, Shimono M, Inoue T. Pulp cell responses during hypoxia and reoxygenation in vitro. European Journal of Oral Sciences, 111(4), 2003, 332-338.

[12]. Fukuyama Y, Ohta K, Okoshi R, Suehara M, Kizaki H, Nakagawa K. Hypoxia induces expression and activation of AMPK in rat dental pulp cells. Journal of Dental Research, 86(9), 2007, 903-907.

[13]. Ueno Y, Kitamura C, Terashita M, Nishihara T. Re-oxygenation improves hypoxia-induced pulp cell arrest. Journal of Dental Research, 85 (9), 2006, 824-828.

[14]. Wang J, Wei X, Ling J, Huang Y, Gong Q. Side population increase after simulated transient ischemia in human dental pulp cell. Journal of Endodontics, 36 (3), 2010, 453-458.

[15]. Ivanovic, Z. Hypoxia or in situ normoxia: The stem cell paradigm. J. Cell. Physiol. 219 (2), 2009, $271-275$.

[16]. Grayson W.L, Zhao F, Izadpanah R, Bunnell B, Ma T. Effects of hypoxia on human mesenchymal stem cell expansion and plasticity in 3D constructs. J. Cell. Physiol. 207 (2), 2006, 331-339.

[17]. Fehrer C, Brunauer R, Laschober G, Unterluggauer H, Reitinger S, Kloss F, Gully C, Gassner R, Lepperdinger G. Reduced oxygen tension attenuates differentiation capacity of human mesenchymal stem cells and prolongs their lifespan. Aging Cell, 6(6), 2007, 745-757.

[18]. Iida K, Takeda-Kawaguchi T, Tezuka Y, Kunisada T, Shibata T, Tezuka K. Hypoxia enhances colony formation and proliferation but inhibits differentiation of human dental pulp cells. Arch Oral Biol. 55(9), 2010, 648-54.

[19]. Kakkar A, Mohanty S, Bhargava B, Airan B. Role of human cardiac biopsy derived conditioned media in modulating bone marrow derived mesenchymal stem cells toward cardiomyocyte-like cells. J Pract Cardiovasc Sci, 1(2), 2015,150-155.

[20]. Pisciotta A, Riccio M, Carnevale G, Beretti F, Maraldi T, Cavallini GM, Ferrari A, Bruzzesi G, De Pol A. Human serum promotes osteogenic differentiation of human dental pulp stem cells in vitro and in vivo. PLoS One, 7(11), 2012,e50542.

[21]. Cooper PD, Burt AM, Wilson JN. Critical Effect of Oxygen Tension on Rate of Growth of Animal Cells in continuous Suspended Cultures. Nature, 182(4648), 1958, 1508-1509.

[22]. Agha-Hosseini F, Jahani MA, Jahani M, Mirzaii-Dizgah I, Ali-Moghaddam K. In vitro isolation of stem cells derived from dental pulp. Clin. Transplant., 24(2),2010, E23-E28.

[23]. Zhou Y, Fan W, Xiao Y. The Effect of Hypoxia on the Stemness and Differentiation Capacity of PDLC and DPC. BioMed Res Int., 2014, 2014:890675.

[24]. Govindasamy V, Ronald VR, Totey S, Din SB, Mustafa WMBW, Totey S, Zakaria Z and Bhonde RR. Micromanipulation of culture niche permits long-term expansion of dental pulp stem cells-an economic and commercial angle. In Vitro Cell Dev Biol Anim., 46(9), 2010, 764-773.

[25]. Lizier N.F, K Alexandre, Gomes C.M., Hebling J, Oliveira C. F, Caplan A.I, Kerkis I. Scaling-Up of Dental Pulp Stem Cells Isolated from Multiple Niches. PLoS One, 7(6), 2012: e39885.

[26]. Hung SP, Ho JH, V. Shih YR, Lo T, L OK. Hypoxia Promotes Proliferation and Osteogenic Differentiation Potentials of Mesenchymal Stem Cells. J Orthod Res, 30(2), 2012,260-266.

[27]. Dominici M, Blanc KL, Mueller I, Slaper-Cortenbach I, Marini F, Krause D, Deans R, Keating A, Prockop D, Horwitz E. Minimal criteria for defining multipotent mesenchymal stromal cells. Cytotherapy, 8(4), 2006, 315-317.

[28]. Eslaminejad MB, Nazarian H, Shariati M, Vahabi S, Falahi F. Isolation and in vitro characterization of Mesenchymal Stem Cells Derived from the Pulp Tissue of Human Third Molar Tooth. Iran J Med Sci, 35(3), 2010, 216-225.

[29]. Holzwrth C, Vaegler M, Gieseke F, Pfister SM, Handgretinger R, Kerst G, Müller I. Low physiologic tensions reduce proliferation and differentiation of human multipotent mesenchymal stromal cells. BMC Cell Bio, 11:11, 2010.

[30]. Ranera B, Remacha AR, Alvarez-Arguedas S, Romero A, Vazquez FJ, Zaragoza P, Martín-Burriel I, Rodellar C. Effect of hypoxia on equine mesenchymal stem cells derived from bone marrow and adipose tissue. BMC Vet Res, 22:8, 2012, 142

[31]. Iida K, Kawaguchi TT, Tezuka Y, Kunisada T, Shibata T, Tezuka K. Hypoxia enhances colony formation and proliferation but inhibits differentiation of human dental pulp cells. Arch Oral Biol, 55(9), 2010, 648-654.

[32]. Malladi P, Xu Y, Chiou M, Giacci A.J., Longaker M.T. . Effect of reduced oxygen tension on chondrogenesis and osteogenesis in adipose derived mesenchymal cells. Am J Physio Cell Physiol., 290(4), 2006,C1139-1146.

[33]. Yamamoto Y, Fujita M, Tanaka Y, Kojima I, Kanatani Y, Ishihara M, \& Tachibana S. Low Oxygen Tension Enhances Proliferation And Maintains Stemness Of Adipose Tissue-Derived Stromal Cells. Biores Open Acces., 2(3), $2013,199-205$.

[34]. Shang J, Liu H, Li J, Zhou Y. Roles of Hypoxia during the chondrogenic differentiation of mesenchymal stem cells. Curr Stem Cell Res Ther., 9(2), 2014,141-7.

[35]. Gronthos S, Brahim J, Li W, Fisher LW, Cherman N, Boyde A, DenBesten P, Robey PG, Shi S. Stem cell properties of human dental pulp stem cells. J Dent Res.,81(8), 2002, 531-535.

[36]. Pfander D, Cramer T, Schipani E, and Johnson RS. HIF-1 $\alpha$ controls extracellular matrix synthesis by epiphyseal chondrocytes. J Cell Sci 116, (Pt9), 2003, 1819-1826. 